\title{
New clues on the interior of Titan from its rotation state
}

\author{
Benoît Noyelles ${ }^{1}$ and Francis Nimmo ${ }^{2}$ \\ ${ }^{1}$ NAmur Center for CompleX SYStems (naXys), University of Namur, Rempart de la Vierge 8, \\ B-5000 Namur, Belgium \\ email: benoit.noyelles@gmail.com \\ ${ }^{2}$ Department of Earth and Planetary Sciences, University of California at Santa Cruz, 1156 \\ High Street, Santa Cruz, California 95064, USA \\ email: fnimmo@es.ucsc.edu
}

\begin{abstract}
The Saturnian satellite Titan is one of the main targets of the Cassini-Huygens mission, which revealed in particular Titan's shape, gravity field, and rotation state. The shape and gravity field suggest that Titan is not in hydrostatic equilibrium, that it has a global subsurface ocean, and that its ice shell is both rigid (at tidal periods) and of variable thickness. The rotational state of Titan consists of an expected synchronous rotation rate and an unexpectedly high obliquity $\left(0.3^{\circ}\right)$ explained by Baland et al. $(2011)$ to be a resonant behavior. We here combine a realistic model of the ice shell and interior and a 6-degrees of freedom rotational model, in which the librations, obliquity and polar motion of the rigid core and of the shell are modelled, to constrain the structure of Titan from the observations. We consider the gravitational pull of Saturn on the 2 rigid layers, the gravitational coupling between them, and the pressure coupling at the liquid-solid interfaces.

We confirm the influence of the resonance found by Baland et al. that affects between 10 and $13 \%$ of the possible Titans. It is due to the 29.5 -year periodic annual forcing. The resonant Titans can be obtained in situations in which a mass anomaly at the shell-ocean boundary (bottom loading) is from 80 to $92 \%$ compensated. This suggests a 250 to $280 \mathrm{~km}$ thick ocean below a 130 to $140 \mathrm{~km}$ thick shell, and is consistent with the degree-3 analysis of Hemingway et al. (2013).
\end{abstract}

Keywords. celestial mechanics, planets and satellites: individual: Titan

The Cassini-Huygens space mission has significantly improved our knowledge of Titan. In particular, there are more and more clues suggesting the presence of a global internal ocean. This contribution aims at modeling the rotation of Titan in considering a rigid shell surrounding a global ocean and a rigid core. We here extend the studies by Baland et al. (2011) and Baland et al. (2014) to a system having 6 dynamical degrees of freedom, i.e. three each (longitude, obliquity and polar motion) for the shell and the inner core.

\section{Our knowledge of Titan}

Several studies since Lunine \& Stevenson (1987) have suggested that Titan's large size results in a global ocean beneath its icy shell. This ocean is consistent with the high tidal Love number measured by Cassini (Iess et al. (2012)).

Hints on the interior can be obtained from the gravity field of Titan that depends on the mass distribution. Unfortunately, two methods of solving for the gravity field do not exactly converge (Tab.1). While the SOL2 solution, using all available data on Titan, could be consistent with a hydrostatic body $\left(J_{2} / C_{22}\right.$ close to $\left.10 / 3\right)$, the SOL1 solution, based only on 4 flybys of Cassini, is inconsistent with hydrostatic equilibrium. Anyway, 
Table 1. The two solutions for the gravity field of Titan, from Iess et al. (2010).

\begin{tabular}{l|r|r|}
\hline & SOL1 & SOL2 \\
\hline$J_{2}$ & $(3.1808 \pm 0.0404) \times 10^{-5}$ & $(3.3462 \pm 0.0632) \times 10^{-5}$ \\
$C_{22}$ & $(9.983 \pm 0.039) \times 10^{-6}$ & $(1.0022 \pm 0.0071) \times 10^{-5}$ \\
$J_{2} / C_{22}$ & $3.186 \pm 0.042$ & $3.339 \pm 0.067$ \\
\hline
\end{tabular}

neither of these two solutions is consistent with a hydrostatic Titan when compared to the shape (Zebker et al. (2009)). This discrepancy led to the suggestion of mass anomalies in Titan, resulting from either lateral variations of the shell thickness (Nimmo \& Bills (2010)) or of the shell density (Choukroun \& Sotin (2012)). The recent degree-3 analysis of the topography by Hemingway et al. (2013) supports lateral variations.

Observations of the rotation of Titan suggest that it rotates synchronously (Meriggiola \& Iess (2012)), as usually expected for the main natural satellites. However, an unexpectedly high obliquity of $18.6 \pm 3$ arcmin at the mean date March $11^{t h}$, 2007 has been measured (Stiles et al. (2008), Meriggiola \& Iess (2012)), that cannot be explained with a rigid Titan (Bills \& Nimmo (2008)). Baland et al. (2011) have shown that the presence of a global ocean could explain this obliquity.

\section{Our model}

We consider Titan as a triaxial body composed of 3 layers: a rigid inner core, a global ocean, and a shell that is completely rigid at tidal periods but deformable at much longer periods, as appropriate for a viscoelastic material like ice. We then build realistic modelTitans. For that, we start from a hydrostatic Titan in which we add a mass anomaly, either at the surface (top loading), or at the ocean-crust boundary (bottom loading). Because the duration of loading is likely tens of Myr at least, we then consider a partial isostatic compensation of this mass anomaly. We assume Airy isostasy, in which lateral variations of the shell thickness occur. Only model Titans that are consistent with the measured shape and gravity fields are kept. Since two solutions exist for the gravity field, we have two sets of model Titans. The resulting model Titans depend on 12 parameters, i.e. a density and 3 radii defining the triaxial outer boundary of each of the three layers. We can reproduce the observations only with bottom loading, and require between 80 and $85 \%$ isostatic compensation for the gravity field SOL1, and between 85 and $92 \%$ for the gravity field SOL2.

We then simulate the rotation of each of these model Titans at the dynamical equilibrium, i.e. synchronous rotation of the two rigid layers and equilibrium obliquity corresponding to the Cassini State 1 for rigid bodies. As Baland et al. (2011) did, we consider the gravitational torque of Saturn on Titan, the gravitational coupling between the two rigid layers, and the pressure coupling at the fluid-solid interfaces. We extended their equations to 6 degrees of freedom, simulating simultaneously, for the two rigid layers, the longitudinal motion, the obliquity, and the polar motion, as Szeto \& Xu (1997) did but without considering the pressure coupling at the two rigid-fluid boundaries. We then extracted the rotational outputs, and in this study we particularly focus on the obliquity. A weakness in our physical model is that we assume that the shell is completely rigid at tidal periods. The elasticity can be critical for the longitudinal librations (Goldreich \& Mitchell (2010), Van Hoolst et al. (2013), Richard et al. (2014)); the extent to which it matters for obliquity is not yet clear.

We model the orbit of Titan around Saturn with TASS1.6 ephemerides (Vienne \& Duriez (1995)), that have the advantage of being available over 9000 years. This is re- 
Table 2. Quasiperiodic decomposition of the orbital quantity $I \exp \imath \Omega$ from TASS1.6 (Vienne $\&$ Duriez (1995)), $I$ being the inclination of Titan and $\Omega$ the longitude of its ascending node. The variations of the shell obliquity resonate with the annual forcing (Term 5).

\begin{tabular}{|r|r|r|c|}
\hline $\mathbf{N}$ & $\begin{array}{r}\text { A mplitude } \\
\text { (arcmin) }\end{array}$ & $\begin{array}{r}\text { Period } \\
(\mathrm{yr})\end{array}$ & Cause \\
\hline 1 & 38.52 & $\infty$ & Sun \\
2 & 19.18 & -703.51 & Saturn's $J_{2}$ \\
3 & 0.90 & -3263.07 & Iapetus \\
4 & 0.77 & 14.73 & Semi-annual \\
5 & 0.13 & -29.46 & Annual \\
\hline
\end{tabular}
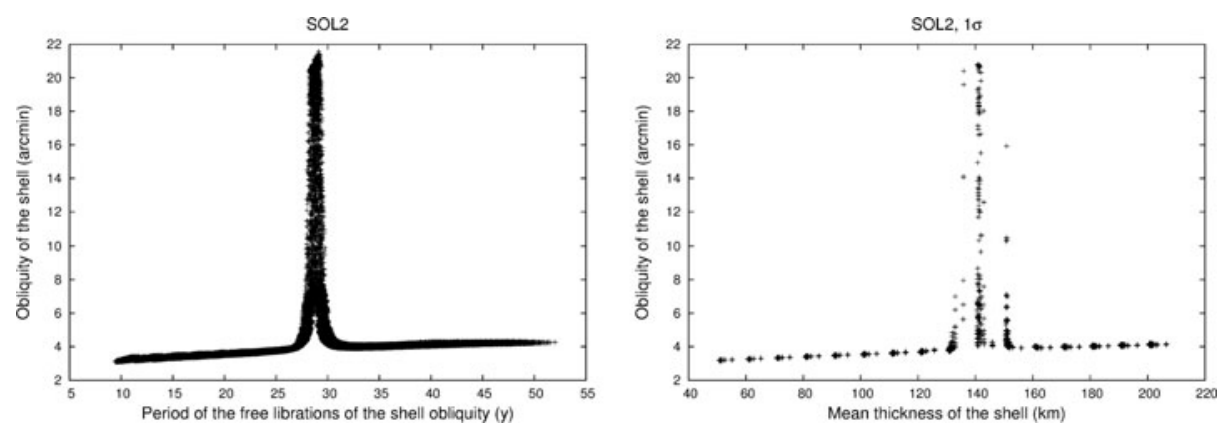

Figure 1. Mean obliquity of Titan vs. the period of the free oscillations (left) and the shell thickness (right). This illustrates how the resonance constrains the interior.

quired to accurately study the obliquity, which results in a slow motion.

\section{A resonant behavior}

Fig.1 shows that two regimes can be expected for the shell obliquity. Away from the peak, we see a small obliquity, close to the one to be expected for a rigid body. However, we see near the peak much larger obliquities. As Baland et al. (2011) already noticed, these larger obliquities are in fact due to a resonance between the annual forcing (see Tab.2) and the free oscillations of the obliquity of the shell, their period being denoted $T_{4}$. Baland et al. (2011) also suggest a possible resonance with the Term 2 of Tab.2 with a wider range of parameters than we have. They also find it in Baland et al. (2014) with a non-hydrostatic Titan and consider it more probable than a resonance with the annual forcing.

For model Titans consistent with the gravity solution SOL1, the high obliquity suggests a shell thickness of $\approx 130 \mathrm{~km}$, and $\approx 140 \mathrm{~km}$ for SOL2. 10 to $13 \%$ of our model Titans fall into this resonance, making it an acceptable explanation.

To deduce the period of the free oscillations $T_{4}$ consistent with the obliquity measurement, we have to keep in mind that the measured obliquity is an instantaneous value, different from the mean obliquity (see Fig. 2). Actually two periods are possible, we have either $T_{4}=29.3 \pm 0.03$ years, or $T_{4}=29.572_{-0.015}^{+0.019}$ years.

\section{A time-varying obliquity}

A numerical simulation of the time evolution of this obliquity predicts a variation of 7 arcmin over the duration of the Cassini mission. The slope should be either positive or 

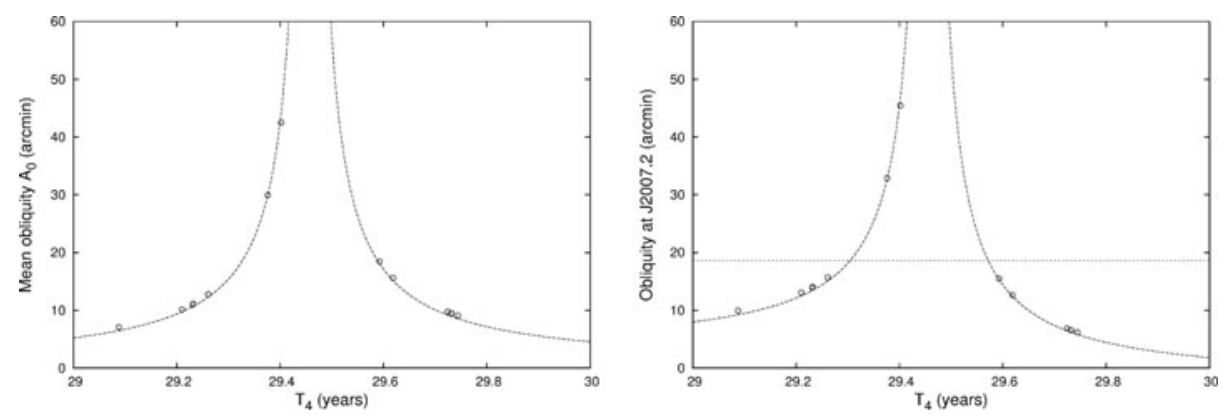

Figure 2. Mean (left) and instantaneous (right) obliquity of Titan. The asymmmetry present for the instantaneous obliquity but absent for the mean one illustrates the difference between the two quantities. The measurement corresponds to the instantaneous obliquity.
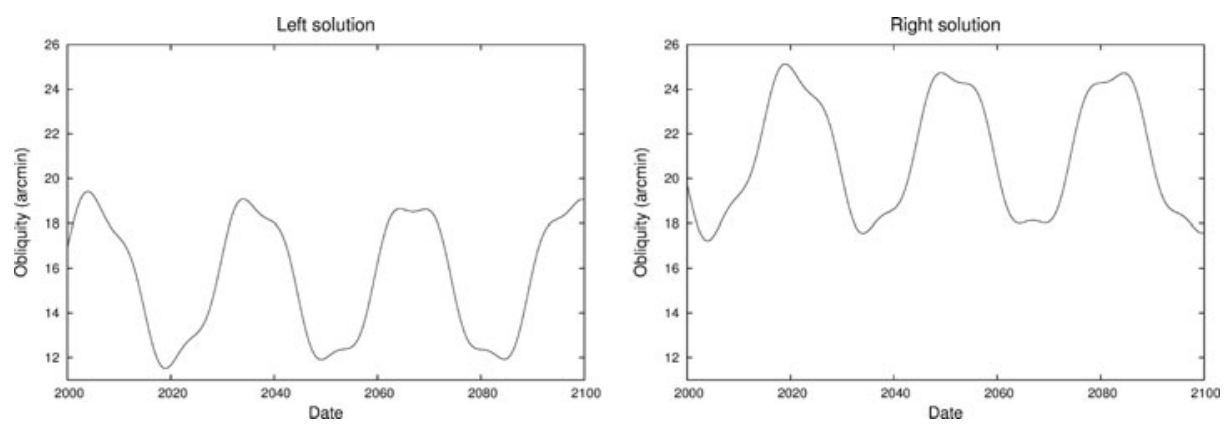

Figure 3. Possible time variations of the obliquity of Titan, with $T_{4}=29.3$ years (left) and $T_{4}=29.572$ years (right).

negative, depending whether $T_{4}$ is smaller or bigger than 29.46 years. So, observing this slope would give an additional constraint on the state of the interior.

\section{References}

Baland, R.-M., Van Hoolst, T., Yseboodt, M., \& Karatekin, Ö. 2011, A\&A, 530, A141

Baland, R.-M., Tobie, G., Lefèvre A., \& Van Hoolst, T., 2014, Icarus, 237, 29

Bills, B. G. \& Nimmo, F. 2008, Icarus, 196, 293

Choukroun, M. \& Sotin, C. 2012, Geophysical Research Letters, 39, L04201

Goldreich, P. M. \& Mitchell, J. L. 2010, Icarus, 209, 631

Hemingway, D., Nimmo, F., Zebker, H., \& Iess, L. 2013, Nature, 500, 550

Iess, L., Rappaport, N. J., \& Jacobson, R. A. et al. 2010, Science, 327, 1367

Iess, L., Jacobson, R. A., \& Ducci, M. et al. 2012, Science, 337, 457

Lunine, J. I. \& Stevenson, D. J. 1987, Icarus, 70, 61

Meriggiola, R. \& Iess, L. 2012, European Planetary Science Congress, id. EPSC2012-593

Nimmo, F. \& Bills, B. G. 2010, Icarus, 208, 896

Noyelles, B., \& Nimmo, F., submitted

Richard, A., Rambaux, N., \& Charnay, B. 2014, Planetary and Space Science, 93-94, 22

Stiles, B. W., Kirk, R. L., \& Lorenz, R. D. et al. 2008, AJ, 135, 1669

Szeto, A. M. K. \& Xu, S. 1997, Journal of Geophysical Research, 102, 27651

Van Hoolst, T., Baland, R.-M., \& Trinh, A. 2013, Icarus, 226, 299

Vienne, A. \& Duriez, L. 1995, A\&A, 297, 588

Zebker, H. A., Stiles, B., \& Hensley, S. et al. 2009, Science, 324, 921 